Purdue University

Purdue e-Pubs

CTRC Research Publications

Cooling Technologies Research Center

$2-21-2008$

Energy Minimization-Based Analysis of Electrowetting for Microelectronics Cooling Applications

Vaibhav Bahadur

Purdue University - Main Campus, vbahadur@purdue.edu

S V. Garimella

Purdue University, sureshg@purdue.edu

Follow this and additional works at: http:/ / docs.lib.purdue.edu/coolingpubs

Bahadur, Vaibhav and Garimella, S V., "Energy Minimization-Based Analysis of Electrowetting for Microelectronics Cooling Applications" (2008). CTRC Research Publications. Paper 93.

http://dx.doi.org/10.1016/j.mejo.2007.11.010

This document has been made available through Purdue e-Pubs, a service of the Purdue University Libraries. Please contact epubs@purdue.edu for additional information. 


\title{
Energy minimization-based analysis of electrowetting for microelectronics cooling applications*
}

\author{
V. Bahadur and S. V. Garimella ${ }^{\dagger}$ \\ School of Mechanical Engineering and Birck Nanotechnology Center, Purdue University, \\ West Lafayette, Indiana 47907, USA
}

\begin{abstract}
Electrowetting(EW)-induced droplet motion has been studied over the last decade in view of its promising applications in the field of microfluidics. The objective of the present work is to analyze the physics underlying two specific EW-based applications for microelectronics thermal management. The first of these involves heat absorption by liquid droplets moving on the surface of a chip under EW actuation. Droplet motion between two flat plates under the influence of an electrowetting voltage is analyzed. An energy minimization framework is employed to predict the actuation force on a droplet. This framework, in combination with semi-analytical models for the forces opposing droplet motion, is used to develop a model that predicts transient EW-induced droplet motion. The second application is targeted at hot spot thermal management and relies on the control of droplet states on artificially structured surfaces through an applied EW voltage. The influence of an electrowetting voltage in determining and altering the state of a static droplet resting on a rough surface is

\footnotetext{
${ }^{*}$ Revised draft for publication in Microelectronics Journal

${ }^{\dagger}$ Corresponding author: E-mail: sureshg@ $@$ purdue.edu
} 
analyzed. An energy-minimization-based modeling approach reveals the influence of interfacial energies, surface roughness parameters and electric fields in determining the apparent contact angle of a droplet in the Cassie and Wenzel states under the influence of an EW voltage. The model is used to establish preliminary criteria to design rough surfaces for use in the hot-spot mitigation application. The concept of an electrically tunable thermal resistance switch for hot-spot cooling applications is introduced and analyzed. Keywords: electrowetting, droplet movement, contact angle, Cassie, Wenzel

\section{Introduction}

Surface tension-based control of fluid motion at the microscale is attractive for a variety of microfluidic applications because of the dominant influence of surface tension at these length scales and the elimination of mechanical moving parts. A number of surface tension-based fluid handling techniques have been discussed in the literature, the most significant among which include electrowetting (EW) [1] (reduction of interfacial tension by the application of an electric field), thermocapillarity driven pumping [2] (reduction of surface tension by temperature, leading to fluid motion) opto-electrowetting-based [3] pumping (lightcontrolled surface tension-induced pumping) and vapor bubble-based pumping [4]. Electrowetting (EW) is a surface tension control-based technique and has been used to demonstrate droplet actuation [5-12] and other microfluidic operations such as the formation, mixing and splitting of droplets [13]. Bahadur and Garimella [14] developed a generic energy minimization-based modeling framework for EW systems and used it to estimate the 
EW actuation force on a droplet moving between two flat plates. The actuation force model was combined with semi-analytical models for predicting the forces opposing droplet motion to develop a model to predict transient EW-induced droplet motion. The first objective of the present work is to highlight the electromechanical nature of electrowetting-induced droplet motion. It is shown that the actuation force predicted by using the concept of an interfacial energy change as described by Bahadur and Garimella [14] is the same as that obtained from a purely electromechanical approach.

The heat dissipation enabled by EW-induced droplet movement offers enormous potential for microelectronics cooling applications. Paik et al. [11] experimentally demonstrated that EW can be used for hot-spot cooling by measuring the decrease in hotspot temperature resulting from EW-induced motion of an aqueous droplet on a flat surface. Electrowetting can also be used to provide hot-spot thermal management solutions through the use of rough surfaces in conjunction with EW. This cooling scheme would involve the use of specially designed rough surfaces to minimize the droplet-substrate contact area while the droplet is being moved to the hot spot by EW-induced actuation. This would result in lower frictional resistance (lower EW voltages) and reduced pre-heating of the droplet in this phase. An additional EW voltage applied at the hot spot would then cause the droplet to spread out and also wet the grooves of the rough surface, thereby enhancing heat transfer. Krupenkin et al. [15] demonstrated such a wetting transition from hydrophobicity to complete wetting in low-surface-tension liquids on nanostructured surfaces under the application of an EW potential. Bahadur and Garimella [16] developed an energy 
minimization based modeling approach to analyze the influence of an electrowetting voltage in determining and altering the state of a static droplet resting on a rough surface. In the present work, an energy minimization framework is utilized to analyze the effects of interfacial tensions, EW fields and surface morphology, and hence to predict wetting behavior on artificially roughened surfaces. The model is then used to establish preliminary design criteria for rough surfaces to be used in the proposed hot-spot cooling scheme. A discussion on the nature, design and fabrication of the rough surfaces is also presented along with proof-of-concept experimental results.

\section{Analysis of EW-induced droplet motion}

The traditional concept of EWOD (ElectroWetting on Dielectric) is based on a reduction in the dielectric-liquid interfacial energy by the application of a voltage between a conducting droplet and an underlying dielectric layer. This reduction in dielectric-liquid interfacial energy $\gamma_{S L}$ upon the application of a voltage $V$ is given by Lippman's equation [17] as:

$\gamma_{S L}=\gamma_{S L}^{0}-\frac{k \varepsilon_{0} V^{2}}{2 d}$

where $\gamma_{S L}^{0}$ is the dielectric-liquid interfacial energy in the absence of an EW voltage and $k$ and $d$ are the dielectric constant and thickness of the dielectric layer. To actuate droplets, the interfacial energy at one end of the droplet is reduced by applying a voltage to an electrode on that end. The electric field-induced reduction in interfacial energy causes the droplet to 
spread locally. The resulting change in contact angles sets up a pressure gradient which drives the droplet towards the actuated electrode. Alternatively, EW-induced droplet motion can also be analyzed using energy-minimization considerations, according to which the droplet minimizes its surface energy by transiting to the actuated electrode. This principle is used in the present work to estimate the actuation force on the droplet as it transitions to the actuated electrode.

\subsection{Physical model}

A device for effecting droplet movement [5] using EW is illustrated schematically in Figure 1. The device shown consists of two flat plates separated by a selected spacing. Control electrodes are fabricated on the lower plate and are individually addressable. They are covered by a dielectric layer and a thin hydrophobic layer to ensure high initial droplet contact angles. The upper plate consists of a single, grounded electrode plane. The top electrode is also coated with a dielectric layer and a hydrophobic layer. The droplet size is chosen to be slightly larger than the electrode pitch so that it overlaps more than one electrode. When a voltage is applied to the electrode on the right on the bottom plate, the dielectric-liquid interfacial tension on the right end of the droplet decreases. The droplet moves towards the energized electrode as a result, and reaches equilibrium when it is at the center of the energized electrode where the surface energy of the droplet is minimized. By pulsing voltages along an array of discrete electrodes, the droplet can be moved continually. 
It is important to note that the droplet is electrically conducting and is typically surrounded by a (non-evaporating) filler fluid such as silicone oil [5] to minimize evaporation.

Figure 1. Electrowetting-induced droplet transport device [14].

The EW-induced actuation force on a droplet can be modeled by estimating the system energy as a function of the droplet position. Figures $2 \mathrm{a}$ and $2 \mathrm{~b}$ show side and top views of an idealized droplet transition, in which the position of the droplet leading edge is tracked by the coordinate $x$. The droplet is sandwiched between two plates with a separation $d$ as shown in Figure 2a with the electrode width set equal to the droplet diameter. A voltage $V$ applied to the right electrode on the bottom plate initiates the transition. The left electrode on the bottom plate and the top plate electrode are grounded. The droplet is assumed to move as a rigid body, maintaining its circular shape during transition. The total system energy consists of the energy stored in the dielectric layers on the top and bottom plates and the energy stored in the filler fluid. The droplet areas covering the actuated electrode $A_{l}$ and the ground electrode $A_{2}$ can be estimated from the droplet geometry.

Figure 2. Schematic of a droplet undergoing transition: (a) side view, and (b) top view [14].

All the capacitances corresponding to the dielectric layers can be represented as parallelplate capacitors (Figure 2a) which form the capacitive network shown in Figure 3. The 
electric field lines in all the parallel-plate capacitors are assumed to be perfectly straight and fringing effects at the sides are not accounted for in the present model. The droplet is assumed to be perfectly conducting; consequently there is no voltage drop across the droplet.

Figure 3. Capacitive network for the estimation of equivalent capacitance of the system.

\subsection{EW-induced actuation force using an electromechanical approach}

The physical model outlined above is set into an analytical framework in this section to estimate the actuation force on a circular droplet during transition. For a droplet of radius $r$ that maintains a circular shape during the entire transition, the areas of the three parallelplate capacitors are given by:

$A_{1}(x)=r^{2} \cos ^{-1}\left(1-\frac{x}{r}\right)-(r-x) \sqrt{r^{2}-(x-r)^{2}}$

$A_{2}(x)=\pi r^{2}-A_{1}$

$A_{3}(x)=\pi r^{2}$

The capacitances of the three parallel-plate capacitors are

$C_{1}(x)=\frac{k_{1} A_{1}(x) \varepsilon_{0}}{d_{1}}$
$C_{2}(x)=\frac{k_{1} A_{2}(x) \varepsilon_{0}}{d_{1}}$ 
$C_{3}(x)=\frac{k_{2} A_{3}(x) \varepsilon_{0}}{d_{2}}$

where $\left(k_{1}, d_{1}\right)$ and $\left(k_{2}, d_{2}\right)$ are (dielectric constant, thickness) of the lower and upper dielectric layers, respectively (Figure $3 \mathrm{a}$ ), $C_{1}$ is the capacitance of the lower plate dielectric with area $\mathrm{A}_{1}, C_{2}$ is the capacitance of the lower plate dielectric with area $A_{2}$, and $C_{3}$ is the capacitance of the upper plate dielectric (Figure 3a). From the circuit in Figure 3, the total lumped capacitance of the system can be expressed as:

$C_{e q}(x)=\frac{C_{1}\left(C_{2}+C_{3}\right)}{\left(C_{1}+C_{2}+C_{3}\right)}$

The energy stored in the capacitive system can then be expressed as a function of the transition position of the droplet as:

$E(x)=\frac{1}{2} C_{e q}(x) V^{2}=\frac{1}{2} \frac{C_{1}\left(C_{2}+C_{3}\right)}{\left(C_{1}+C_{2}+C_{3}\right)} V^{2}$

The derivative of this energy variation gives the electrical actuation force on the droplet as a function of the transition position $x$ as:

$$
F_{a c t}(x)=\frac{1}{2} \frac{\left(C_{2}+C_{3}-C_{1}\right)}{\left(C_{1}+C_{2}+C_{3}\right)} \frac{d C_{1}}{d x} V^{2}
$$

The actuation force obtained from Equation 10 using a purely electromechanical approach is identical to the actuation force obtained by employing Lippman's equation by Bahadur and Garimella [14]. This emphasizes the electromechanical nature of electrowetting-induced droplet pumping since the EW-induced reduction of dielectric-liquid interfacial tension, 
implied by Lippman's equation, is never used in arriving at Equation 10. Alternatively, the Lippman equation-based (Equation 1) reduction of dielectric-liquid interfacial tension can itself be considered as having electromechanical origins; consequently use of Lippman's equation leads to the same actuation force results as those from an electromechanical model.

All the actuation force results arrived at in [14] can be reproduced using the electromechanical approach outlined above. Most significantly, it can be shown that for the same dielectric material, the top plate dielectric layer must be thinner than the bottom plate dielectric layer (i.e., $d_{2} / d_{1}<1$ ) to ensure a positive actuation force during the entire transition. Furthermore, the actuation force on the droplet at all stages during transition is maximized when the top dielectric layer is absent; however, a top-plate dielectric is often necessitated to avoid chemical reactions in the droplet. This maximum actuation force in the absence of a top dielectric layer can be derived as:

$$
F_{\text {max }}(x)=\frac{1}{2} \frac{d C_{1}}{d x} V^{2}
$$

\subsection{Modeling EW-induced droplet motion}

The dominant forces [14] opposing EW-induced droplet motion are the shear forces due to the top and bottom plates, the viscous drag exerted by the filler fluid, and the contact-line friction force. Significant challenges exist in modeling each of these force components; however, the present work is not aimed at resolving these challenges. Semi-empirical expressions are therefore used to model the opposing forces as shown in Reference 14. 
The overall differential equation governing EW-induced droplet motion is then given as:

$m \frac{d^{2} x}{d t^{2}}=F_{a c t}-\left(\frac{6 \mu_{l} v}{d}\right)\left(2 \pi r^{2}\right)-\left(\frac{1}{2} C \rho_{f} v^{2}\right)(2 r d)-(\zeta v)(4 \pi r)$

where $m$ is the droplet mass. The first opposing force on the right hand side of Equation 12 is the viscous force on the droplet due to the top and bottom plates ( $v$ is the droplet velocity and $\mu_{l}$ is the droplet viscosity). The second opposing force on the right hand side of Equation 12 is the viscous stress due to the filler fluid, which is estimated by considering the droplet as a rigid body moving through the filler fluid $(C$ is the drag coefficient for a cylinder in cross flow and $\rho_{f}$ is the filler fluid density). The third opposing force on the right hand side of Equation 12 is the contact-line friction force, which is assumed to be

proportional to the droplet velocity as per the approach of Ren et al. [5]. ( $\zeta$ is a proportionality coefficient with a value $\left.\zeta=0.04 \mathrm{Ns} / \mathrm{m}^{2}[5]\right)$

\subsection{Droplet movement results}

The above differential equation (Equation 12) can be solved to obtain the droplet position and velocity variation with time. Figure 4 shows the transition time required for a $1 \mathrm{~mm}$ radius water droplet to move to the actuated electrode as a function of the applied voltage for three plate spacings. (The actuation force is obtained for the no-top-plate dielectric condition which ensures the maximum actuation force during transition. The bottom plate has a $1 \mu \mathrm{m}$ thick dielectric layer with a dielectric constant of 3). From Figure 4, it is seen that droplet transition times strongly depend on the actuation voltage, which underscores the dominant 
influence of the voltage on the actuation force. No significant dependence of transition time on the gap spacing is seen, except in the low-voltage, low-velocity regime where a slight decrease in transition time is observed with increased gap spacing. While an increase in gap spacing leads to an increased droplet mass, the opposing viscous forces also change with gap spacing. The net effect is the absence of any substantial dependence of transition time on gap spacing. Average and peak velocities of $7 \mathrm{~cm} / \mathrm{s}$ and $10 \mathrm{~cm} / \mathrm{s}$ respectively were obtained for an actuation voltage of $50 \mathrm{~V}$ which are comparable to the experimental results of Pollack et al. [5] and the modeling results of Oprins et al. [12].

Figure 4. Droplet transition time dependence on voltage and plate spacing (transition distance is $2 \mathrm{~mm}$ ) [14].

\section{Electrowetting on rough surfaces for hot spot thermal management}

Surface morphology can substantially alter the contact angle of a droplet and effectively reduce the contact area of the droplet with the substrate [18]. EW voltages can be significantly reduced if the surface resistance offered to droplet motion is decreased. EW can be used in conjunction with rough surfaces to develop a hot-spot cooling scheme in which a droplet is directed to a particular hot spot in this low EW voltage, low contact area mode. This is also desirable from a thermal standpoint since the low contact area ensures less pre-heating of the droplet, which maximizes its cooling capacity once it reaches the hot spot. Once at the hot spot, the droplet is spread by an additional EW voltage, resulting in 
intimate contact with the enhanced area offered by the surface features, and hence, enhanced heat transfer. The droplet is then retracted to its hydrophobic state by turning off the additional voltage and pumped away in the low surface resistance mode.

\subsection{Droplet states on rough surfaces}

The design of surfaces for this application first requires an understanding of static droplet behavior on rough surfaces under the application of an EW voltage. The influence of surface roughness is generally understood by a study of two extreme configurations in which a static droplet can rest on a given rough surface. In the first configuration shown in Fig. 5a, the droplet rests entirely on the tips of the rough surface; a droplet exhibiting such composite contact with solid surfaces and the intervening air is referred to as a Cassie droplet [19]. If alternatively, the droplet wets the entire surface including the grooves as shown in Fig. 5b, it is referred to as a Wenzel droplet [20]. He et al. [21] provided experimental evidence that both Cassie and Wenzel droplets are possible on the same surface based on the way the droplets are formed. The Cassie state was achieved by gently depositing the droplet on the rough surface while the Wenzel state was realized by dropping the droplet from a height. Patankar [22] showed that the surface energy of the Cassie and Wenzel droplets is minimized when the apparent (macroscopic) contact angle equals that predicted by the Cassie and Wenzel equations. However, the Cassie and Wenzel droplets differ in their surface energies, and the stable equilibrium state of the droplet corresponds to the state which has a lower energy. The influence of EW on droplet behavior on rough surfaces has been the focus of a few research efforts. Krupenkin et al. [15] demonstrated that a droplet resting on a 
nanostructured surface can be made to transition to a completely wetting (Wenzel) state from an initial hydrophobic (Cassie) state by the application of an EW voltage. Bahadur and Garimella [16] developed an energy-minimization based modeling approach to predict the contact angle in the Cassie and Wenzel states under the influence of an electrowetting voltage.

Figure 5. Droplet states on electrowetted rough surfaces: (a) Cassie state, and (b) Wenzel state.

In the Cassie state, the droplet sits on a composite surface of air and solid. The apparent contact angle $\theta_{C}$ of such a droplet can be obtained [18] from energy-minimization principles as:

$\cos \theta_{C}=-1+\phi\left(1+\cos \theta_{0}\right)$

where $\phi$ is the fraction of the solid contacting the liquid and $\theta_{0}$ is the contact angle of the droplet on a flat surface. The hydrophobicity of a Cassie droplet increases as $\phi$ decreases because of enhanced contact with air. In the Wenzel state, the droplet is in intimate contact with the surface features. For such a droplet the apparent contact angle $\theta_{W}$ is obtained as:

$\cos \theta_{W}=r_{m} \cos \theta_{0}$

where $r_{m}$ is the roughness factor defined as the ratio of the total surface area (including the sides and base of the roughness elements) to the projected surface area (not including the 
sides of the roughness elements). The Wenzel state thus amplifies the initial hydrophobicity or hydrophilicity of the droplet.

\subsection{Droplet states on electrowetted rough surfaces}

An energy-minimization approach can also be used to develop expressions for the apparent contact angle of a droplet resting on a rough surface in the presence of an EW voltage [16]. The rough surface under consideration must be electrically conducting and is coated with a thin dielectric layer of thickness $d$ and dielectric constant $k$ that conforms to the surface roughness features (not shown in Figure 5). An EW field is established across the dielectric by an electrode contacting the conducting droplet and the electrically conducting substrate. The energy-minimization approach consists of estimating the total droplet surface energy as the sum of the solid-liquid, liquid-air and solid-air interfacial energies. EW changes the surface energy content of the droplet by lowering the solid-liquid interfacial energy as predicted by Lippman's equation [17]. Minimization of the surface energy subject to a constant droplet volume constraint gives the apparent contact angle of the droplet on the

electrowetted rough surface. The dimensions of the droplet are such that the effect of gravity is neglected; consequently the droplet shape is assumed to be that of a truncated sphere, and is used in estimating the interfacial areas.

The apparent contact angle of a Cassie droplet under the influence of an EW voltage can be obtained following a procedure similar to that adopted by Patankar [22] for no applied 
voltage. The total surface energy $E$ of a Cassie droplet of radius $R$ and apparent contact angle $\theta$ under the application of an EW voltage $V$ can be expressed as:

$E=\left[A_{L A}+A_{S L}(1-\phi)\right] \gamma_{L A}^{0}+\phi A_{S L}\left(\gamma_{S L}^{0}-\gamma_{S A}^{0}-\frac{k \varepsilon_{0} V^{2}}{2 d}\right)$

where $A_{L A}=2 \pi R^{2}(1-\cos \theta)$

and $A_{S L}=\pi R^{2} \sin ^{2} \theta$

$A_{L A}$ and $A_{S L}$ are the apparent liquid-air and solid-liquid interfacial areas, respectively, and $\gamma_{L A}^{0}, \quad \gamma_{S L}^{0}$ and $\gamma_{S A}^{0}$ are the liquid-air, solid-liquid and solid-air interfacial energies, respectively. At equilibrium, the total surface energy of the droplet is minimized subject to a constant droplet volume constraint:

$V=\frac{\pi R^{3}}{3}(1-\cos \theta)^{2}(2+\cos \theta)$

The method of Lagrange multipliers is employed to solve this constrained minimization problem [22], and results in the following equations:

$\frac{\partial E}{\partial R}=\lambda \frac{\partial V}{\partial R}$

$\frac{\partial E}{\partial \theta}=\lambda \frac{\partial V}{\partial \theta}$

where $\lambda$ is the Lagrange multiplier. Elimination of $\lambda$ from Equations 22 and 23 yields the apparent contact angle of a Cassie drop on an electrowetted surface $\theta_{C}^{E}$ as: 
$\cos \theta_{C}^{E}=-1+\phi\left(1+\frac{\left(\gamma_{S A}^{0}-\gamma_{S L}^{0}\right)}{\gamma_{L A}^{0}}+\eta\right)$

The interfacial energies can be related to the droplet contact angle $\theta_{0}$ on a flat surface by Young's equation:

$\gamma_{S A}^{0}-\gamma_{S L}^{0}=\gamma_{L A}^{0} \cos \theta_{0}$

which yields:

$\cos \theta_{C}^{E}=-1+\phi\left(1+\cos \theta_{0}+\eta\right)$

where $\eta$ is the electrowetting number expressed as:

$\eta=\frac{k \varepsilon_{0} V^{2}}{2 d \gamma_{L A}^{0}}$

A similar procedure can be applied to estimate the apparent contact angle $\theta_{W}^{W}$ of a Wenzel drop under an EW voltage as:

$\cos \theta_{W}^{W}=r_{m}\left(\cos \theta_{0}+\eta\right)$

An EW voltage decreases the apparent contact angle in both the Cassie and Wenzel states, similar to the well-known EW effect on a flat surface. It is seen that the contact angle on a rough surface with an EW voltage (Equations 23 and 25) can be obtained from the equations in the absence of EW (Equations 13 and 14) by replacing $\cos \theta_{0}$ with $\left(\cos \theta_{0}+\eta\right)$. A comparison of the EW number and $\cos \theta_{0}$ therefore gives a direct measure of the relative influence of electrostatic energy and interfacial energies in determining the contact angle of the droplet. 
Patankar [22] showed that the surface energy of a constant-volume droplet can be expressed solely in terms of the apparent contact angle. Furthermore, the droplet surface energy increases with the apparent contact angle of the droplet. This implies that the lower of the Cassie and Wenzel angles corresponds to the stable equilibrium position of the droplet. This principle can be used to design rough surfaces on which the droplet states are manipulated dynamically by changing the relative energy content (and relative stability) of the Cassie and Wenzel states. For instance, a surface can be designed such that the Cassie state is lower in energy than the Wenzel state in the absence of an EW voltage. The droplet can then be made to transition to the Wenzel state by applying an EW voltage, as long as the roughness parameters and the applied EW voltage are such that the electrowetted Wenzel state is lower in energy than the electrowetted Cassie state. EW is thus a powerful tool to manipulate droplet behavior on rough surfaces.

\subsection{Design criteria for rough surfaces for hot-spot cooling}

The relationships developed in section 3.2 can be used to design rough surfaces for the hot-spot cooling application discussed earlier. The requirements of low sliding resistance and low contact area while the droplet is moving to the hot spot dictate that the Cassie state must be lower in energy than the Wenzel state in the absence of an EW voltage. This implies that the Cassie angle $\theta_{C}$ must be lower than the Wenzel angle $\theta_{W}$ or:

$\cos \theta_{C}>\cos \theta_{W}$

Equation 13 and Equation 14 yield two conditions required to satisfy the above restriction: 
$\theta_{0}>90^{\circ}$,

and $\cos \theta_{0}<-\frac{(1-\phi)}{\left(r_{m}-\phi\right)}$

Equation 27 implies that the contact angle of the droplet on a flat surface should be more than $90^{\circ}$ and Equation 28 can be used to decide the roughness $r_{m}$ for a specified $\phi$.

The requirement of increased contact area of the droplet and intimate contact with the surface features on application of an EW voltage implies that the electrowetted Wenzel state must be lower in energy than the electrowetted Cassie state. This implies that:

$$
\cos \theta_{W}^{W}>\cos \theta_{C}^{W},
$$

which leads to:

$$
\eta>-\cos \theta_{0}-\frac{(1-\phi)}{\left(r_{m}-\phi\right)}
$$

Equation 30 can be used to estimate the minimum voltage required to switch from the Cassie state to the Wenzel state. The application of an EW voltage will also lead to a contact angle decrease in both states which further increases the droplet area available for heat transfer. The magnitude of this area enhancement depends on the applied EW voltage, and the contact area increases with voltage until the onset of the contact angle saturation phenomenon [17].

\subsection{Electrowetting-based thermal resistance switch}

The hot-spot cooling application described above can be better understood by treating the Cassie and Wenzel droplet states as the 'off' and 'on' positions of a thermal resistance 
switch. The droplet in its Cassie (off) state offers a high thermal resistance (between the base of the substrate and the base of the droplet) to heat transfer from the substrate to the droplet due to the low contact area and the air gap resulting from the grooves. The thermal resistance in the Wenzel (on) state is dramatically reduced when the contact area is increased and the droplet wets the grooves. This concept of a thermal switch can be examined by calculating the change in thermal resistance to heat transfer to the droplet, resulting from the application of an EW voltage.

One such rough silicon surface (coated with a thin hydrophobic layer) which meets all the design criteria using a water droplet can be described by $\phi=0.1$ and $r_{m}=3$. Assuming an array of $10 \mu \mathrm{m}$ square pillars, the pitch and the height of the pillars can be estimated as $31.6 \mu \mathrm{m}$ and $50 \mu \mathrm{m}$, respectively. A steady-state conduction analysis was carried out to obtain a preliminary estimate of the thermal resistance to heat transfer to the droplet in the on and off states. In the off (Cassie) state the thermal resistance between the droplet base and the substrate was calculated by estimating the thermal conduction resistances of the air gap and the silicon pillars as being in parallel. In the on (Wenzel) state the thermal resistance was calculated by estimating the thermal resistance of the water-filled grooves and the silicon pillars as being in parallel. The reduction in thermal resistance in the on state is due to two factors. The apparent contact angle of the droplet decreases upon the application of an EW voltage and the droplet spreads out, which increases the heat transfer area. The second contribution to the decrease in thermal resistance comes from the filling of the grooves with water instead of air. Conduction calculations reveal that for the surface being 
considered, the thermal resistance decreases by a factor of 9.7 when the droplet switches from the off to the on state. This reduction in thermal resistance is primarily because of the increased heat transfer area as a result of the contact angle reduction. The thermal conductivity of silicon is more than two orders of magnitude higher than air and water;

consequently, filling the grooves with water (which is significantly more conducting than air) does not lead to a significant reduction in thermal resistance, since most of the heat flows through the silicon pillars. For the rough surface being considered, $\theta_{C}=160^{\circ}$ while $\theta_{W}^{W}=111^{\circ}$ for an EW number $\eta=0.3$. This large change in contact angle increases the apparent area for heat transfer by 9.4 times, leading to a significant reduction in thermal resistance. The filling of the grooves would also contribute to significant reduction in thermal resistance in situations where the thermal conductivity of the solid is comparable to that of the droplet fluid.

\subsection{Rough surfaces for the thermal resistance switch application}

Droplet behavior on structured surfaces has been studied for more than five decades and well-characterized fabrication techniques for the creation of artificially structured surfaces are available. Silicon-based rough surfaces required for the thermal resistance switch application can be fabricated by deep reactive ion etching using an electrically conductive silicon substrate, as demonstrated by Krupenkin et al. [15] Advances in thick-film photoresists offer the possibility of fabricating rough surfaces out of thick photoresists as demonstrated by Herbertson et al. [23] who fabricated rough surfaces from SU-8 for 
electrowetting applications. An important consideration in the design of such surfaces concerns the size scale of the roughness elements. Krupenkin et al. [15] fabricated nanostructured surfaces and demonstrated EW-induced Cassie-Wenzel transition on such surfaces. Patankar et al. [21] and Herbertson et al. [23] demonstrated Cassie-Wenzel transitions on surfaces in which the roughness elements were on the micron-scale. The optimum length scale of the roughness elements is not yet fully understood and will also determine the frictional resistance to fluid motion once the droplet penetrates the grooves. Another key issue encountered in preliminary EW experiments on rough surfaces concerns the lack of reversibility between the Cassie and Wenzel states. Krupenkin et al. [15] and Herbertson et al. [23] did not observe the droplet transitioning back to the Cassie state upon the removal of the EW voltage. Krupenkin et al. [24] recently demonstrated reversible transition by passing a heat pulse through the substrate while the droplet was in the Cassie state, thereby evaporating a part of the liquid adjacent to the substrate. The opposing forces inhibiting reversibility need to be further understood, quantified and factored into the surface design to enable reversible surfaces for application as thermal resistance switches.

Experimental investigations of droplet behavior on artificially structured surfaces are underway with the objective of resolving these issues. Figure 6 (on the left) shows a water droplet on a microstructured silicon surface in the absence of an electrowetting voltage. The droplet is clearly in the Cassie state as is evident from the light visible through the silicon pillars. The droplet can be made to transition to the Wenzel state (droplet on the right in Figure 6) upon the application of an EW voltage of $80 \mathrm{~V}$. To the authors' best knowledge, 
this is the first reported direct visual confirmation of droplet state transition induced by an electrowetting voltage. Extensive experimentation is underway to understand and experimentally quantify droplet behavior on artificially structured surfaces.

Figure 6. Droplet states on electrowetted rough surfaces, The droplet on the left is in the Cassie state in the absence of an EW voltage, but transitions to the Wenzel state (shown on the right) at an EW voltage of $80 \mathrm{~V}$.

\section{Conclusions}

An energy minimization-based approach is used to estimate the EW-induced actuation force on a droplet as it transitions to the actuated electrode. It is shown that the actuation force obtained using a purely electromechanical approach is the same as that obtained from the use of interfacial energy change predicted by Lippman's equation. The actuation force model is then combined with semi-empirical models for predicting forces opposing droplet motion to yield a simplified model for predicting transient droplet motion.

The second part of the paper uses the energy minimization principle to develop a theory for predicting the behavior of static droplets on rough electrowetted surfaces. Existing equations for contact-angle prediction on rough surfaces are extended to account for the effects of EW. It is seen that EW is a powerful tool to control droplet behavior on rough surfaces. The model developed is applied to establish basic design criteria for rough surfaces 
for use in a hot-spot cooling application, which works on the principle of an electrically tunable thermal resistance switch. The dramatic switch in thermal resistance resulting from an EW voltage highlights the potential of EW for developing site-specific thermal management solutions.

\section{References}

[1] Kim C J. Micropumping by electrowetting. ASME Int. Mech. Engg. Cong. and Expo. New York IMECE2001/HTD-24200, 2001.

[2] Sammarco T S and Burns M A. Thermocapillary pumping of discrete drops in microfabricated analysis devices. AIChE Journal 1999; 45: 350-366.

[3] Chiou P Y, Moon H, Toshiyoshi H, Kim C J and Wu M C. Light actuation of liquid by optoelectrowetting. Sensors and Actuators A 2003; 104: 222-228.

[4] Geng X, Yuan H, Oguz H N and Prosperetti A. Bubble-based micropump for electrically conducting liquids. Journal of Micromechanics and Microengineering 2001; 11: 270-276.

[5] Pollack M G, Shenderov A D and Fair R B. Electrowetting-based actuation of droplets for integrated microfluidics. Lab on a Chip 2002; 2: 96-101.

[6] Pollack M G and Fair R B. Electrowetting-based actuation of liquid droplets for microfluidic applications. Applied Physics Letters 2000; 77: 1725-1726. 
[7] Mohseni K and Dolatabadi A. Electrowetting droplet actuation in micro scale devices. 43rd AIAA Aerospace Science Meeting and Exhibit Nevada AIAA-677, 2005.

[8] Kuo J S, Spicar-Mihalic P, Rodriguez I and Chiu D T. Electrowetting-induced droplet movement in an immiscible medium. Langmuir 2003; 19: 250-255.

[9] Yi U C and Kim C J. EWOD actuation with electrode-free cover plate. 13th Int. Conf. on Solid-State Sensors, Actuators and Microsystems Seoul (South Korea), 2005.

[10] Torkkeli A, Saarilahti J, Haara A, Harma H, Soukka T and Tolonen P. Electrostatic transportation of water droplets on superhydrophobic surfaces. 14th Intl. IEEE Conference on MEMS 2001; 475-478.

[11] Paik P, Pamula V K and Chakrabarty K. Adaptive hot-spot cooling of integrated circuits using digital microfluidics. Proceedings of IMECE 2005 IMECE2005-81081, Florida (USA), 2005.

[12] Oprins H, Vandevelde B, Beyne E, Borghs G and Baelmans M. Selective cooling of microelectronics using electrostatic actuated liquid droplets-modeling and experiments. International Workshop on Thermal Investigations of ICs and Systems Cote d'Azur (France) 207-212, 2004.

[13] Fair R B, Srinivasan V, Ren H, Paik P, Pamula V K and Pollack M G. Electrowetting-based on-chip sample processing for integrated microfluidics IEEE Inter. Electron Devices Meeting, 2003. 
[14] Bahadur V \& Garimella, S V. An Energy-based Model for Electrowetting-induced Droplet Actuation. Journal of Micromechanics and Microengineering 2006 ; 16: 1494-1503.

[15] Krupenkin T N, Taylor J A, Schneider T M and Yang, S. From Rolling Ball to Complete Wetting: The Dynamic Tuning of Liquids on Nanostructured Surfaces. Langmuir 2004; 20: 3824-3827.

[16] Bahadur V and Garimella, S V. Electrowetting-based control of static droplet states on rough surfaces. Langmuir 2007; 23: 4918-4924.

[17] Mugele F and Baret J C. Electrowetting: from basics to applications. Journal of Physics: Condensed Matter 2005; 17: 705-774.

[18] Quere D. Non-sticking drops. Reports on Progress in Physics 2005; 68: 2495-2532.

[19] Cassie A B D. Contact Angles. Discussions of the Faraday Society 1948: 3: 11-16.

[20] Wenzel T N. Surface Roughness and Contact Angles. Journal of Physical and Colloid Chemistry 1949; 53: 1466-1467.

[21] He B, Patankar N A and Lee J. Multiple Equilibrium Droplet Shapes and Design Criterion for Rough Hydrophobic Surfaces. Langmuir 2003; 19: 4999-5003.

[22] Patankar N A. On the Modeling of Hydrophobic Contact Angles on Rough Surfaces. Langmuir 2003; 19: 1249-1253.

[23] Herbertson D J, Evans R E, Shirtcliffe N J, McHale G and Newton M I. Electrowetting on Superhydrophobic SU-8 Patterned Surfaces. Sensors Actuators A 2006; 130: 189-193. 
[24] Krupenkin T N, Taylor J A, Wang E N, Kolodner P, Hodes M and Salamon T R. Reversible Wetting-Dewetting Transitions on Electrically Tunable Superhydrophobic Nanostructured Surfaces. Langmuir 2007; 23: 9128-9133. 